\title{
SPECTRAL VARIATIONS OF THE SKY: CONSTRAINTS ON ALTERNATE UNIVERSES
}

\author{
R. CHARY ${ }^{1,2,3}$ \\ ${ }^{1}$ Infrared Processing and Analysis Center, MS314-6, California Institute of Technology, Pasadena, CA 91125, USA; rchary@caltech.edu \\ ${ }^{2}$ Smithsonian Astrophysical Observatory, 60 Garden Street, Cambridge, MA 02138, USA \\ Received 2015 September 2; accepted 2015 November 25; published 2016 January 20
}

\begin{abstract}
The fine tuning of parameters required to reproduce our present day universe suggests that our universe may simply be a region within an eternally inflating super-region. Many other regions beyond our observable universe would exist with each such region governed by a different set of physical parameters. Collision between these regions, if they occur, should leave signatures of anisotropy in the cosmic microwave background (CMB) but have not been seen. We analyze the spectral properties of masked, foreground-cleaned maps between 100 and $545 \mathrm{GHz}$ constructed from the Planck data set. Four distinct $\sim 2^{\circ}-4^{\circ}$ regions associated with CMB cold spots show anomalously strong $143 \mathrm{GHz}$ emission but no correspondingly strong emission at either 100 or $217 \mathrm{GHz}$. The signal to noise of this $143 \mathrm{GHz}$ residual emission is at the $\gtrsim 6 \sigma$ level which reduces to $3.2-5.4 \sigma$ after subtraction of remaining synchrotron/free-free foregrounds. We assess different mechanisms for this residual emission and conclude that although there is a $30 \%$ probability that noise fluctuations may cause foregrounds to fall within $3 \sigma$ of the excess, there is less than a $0.5 \%$ probability that foregrounds can explain all the excess. A plausible explanation is that the collision of our universe with an alternate universe whose baryon to photon ratio is a factor of $\sim 4500$ larger than ours, could produce enhanced hydrogen Paschen-series emission at the epoch of recombination. Future spectral mapping and deeper observations at 100 and $217 \mathrm{GHz}$ are needed to mitigate systematics arising from unknown Galactic foregrounds and to confirm this unusual hypothesis.
\end{abstract}

Key words: cosmic background radiation - cosmology: observations - diffuse radiation - surveys

\section{INTRODUCTION}

Following a hot Big Bang, as the universe cooled, the electrons and protons recombined at redshifts between $z \sim 1500$ and 1000. The photons last decoupled from the particles at $z \sim 1050$, at which point the universe became transparent. This process of recombination causes bound-bound transitions of the electrons within the energy shells of hydrogen and helium as well as free-bound transitions, both of which produce line and continuum emission. Detailed modeling of the recombination line emission has been presented and its contribution as distortions to the cosmic microwave background (CMB) spectrum at $\mathrm{GHz}$ frequencies previously calculated (Chluba \& Sunyaev 2006; Rubiño-Martín et al. 2008; Ali-Haïmoud \& Hirata 2011; Ali-Haïmoud 2013). From these redshifts, the recombination line emission of the Balmer and Paschen series is redshifted to the frequency range $100-1000 \mathrm{GHz}$, conveniently observed by the Planck satellite.

The Planck satellite (Planck Collaboration et al. 2014b) was launched on 2009 May 14 and observed the sky stably and continuously from 2009 August 12 to 2013 October 23. Planck's scientific payload included the High Frequency Instrument (HFI; Lamarre et al. 2010) whose bolometers cover bands centered at 100, 143, 217, 353, 545, and $857 \mathrm{GHz}$. Planck imaged the whole sky twice per year, with a combination of sensitivity, angular resolution, and frequency coverage never before achieved. The HFI instrument ran out of liquid helium cryogen in 2012 January which allowed for almost five full sky surveys to be performed. The multiwavelength coverage has allowed separation of the individual components of emission with spectacular precision (Planck Collaboration et al. 2014c, 2014d). However, Planck (and $W M A P)$ are not absolutely calibrated missions, and as such are

\footnotetext{
${ }^{3}$ Visiting Associate.
}

insensitive to spectral distortions of the CMB blackbody, i.e., the monopole, which was exquisitely measured by the FIRAS instrument on COBE (Mather et al. 1994).

The cold spots in the CMB correspond to the deeper potential wells produced by quantum fluctuations (Sachs \& Wolfe 1967). Since the recombination rates in over dense regions of the universe could be higher than those in under dense regions by the clumping factor of the plasma, one can search for the recombination line signature in the CMB cold spots, despite the lack of spectral constraints on the monopole by Planck. By convolving the simulated recombination line spectrum (Chluba \& Sunyaev 2006; Chluba et al. 2007; Rubiño-Martín et al. 2008) through the Planck bandpasses, it is easy to estimate that the spectral distortion due to line emission is several orders of magnitude below the noise threshold of the Planck all sky maps (Table 1). Based on the relative signal-tonoise ratios $(\mathrm{S} / \mathrm{Ns})$, it can be seen that the recombination line signature would be the strongest in the Planck 143 and $353 \mathrm{GHz}$ bands.

The fine structure constant and baryon density of our universe makes it challenging to detect such recombination line emission with the existing data (Table 1). However, if bubbles of alternate universes with physical properties radically different from our own (Aguirre \& Tegmark 2005), intersected in spacetime with our universe, it could potentially result in anisotropies in the CMB temperature fluctuations (Dai et al. 2013) and an enhancement of the recombination line signature making it more observable in existing data. Although temperature anisotropies in the CMB fluctuations have been found to be consistent with an isotropic universe (Planck Collaboration et al. 2015a), these analysis have relied on the spatial statistics of the resultant CMB and have not utilized the spectral variation of the CMB. For example, using WMAP data, it was argued that the number of colliding bubbles is $<1.6$ at 
Table 1

Comparison Between the Expected Hydrogen and Helium Recombination Line Signal in the Planck Bands and the Noise Properties of the Frequency Maps

\begin{tabular}{|c|c|c|c|c|c|c|}
\hline Frequency $(\mathrm{GHz})$ & 100 & 143 & 217 & 353 & 545 & 857 \\
\hline Recombination line signal $\left(10^{-7} \mathrm{MJy} \mathrm{sr}^{-1}\right)$ & 1.59 & 2.79 & 1.69 & 6.52 & 2.37 & 2.15 \\
\hline Median noise in Planck maps ${ }^{\mathrm{a}}\left(10^{-3} \mathrm{MJy} \mathrm{sr}^{-1}\right)$ & 11 & 7.16 & 13.1 & 25.7 & 27.1 & 25.3 \\
\hline $\mathrm{S} / \mathrm{N}\left(10^{-5}\right)$ & 1.45 & 3.89 & 1.29 & 2.53 & 0.872 & 0.851 \\
\hline Noise in deepest part of map ${ }^{\mathrm{a}}\left(10^{-3} \mathrm{MJy} \mathrm{sr}^{-1}\right)$ & 2.56 & 1.74 & 3.47 & 5.24 & 5.23 & 4.97 \\
\hline Area of deepest part of map $\left(\mathrm{deg}^{2}\right)$ & 88 & 105 & 157 & 55 & 41 & 47 \\
\hline
\end{tabular}

Note.

${ }^{\mathrm{a}}$ Noise is measured per 1 '.8 pixel.

$68 \%$ confidence (e.g., Feeney et al. 2011). Here, we attempt to constrain the recombination line signature through spectral analysis of the CMB distortions in the Planck bands and through this analysis, assess the presence of universes with properties that are different from our own. We note that the regions that result from the interaction of our universe with other universes need to be larger than the Hubble horizon at the surface of last scattering; this implies than any such regions would be $\gg 1^{\circ}$.

As has been pointed out by Desjacques et al. (2015), contamination from foreground sources, particularly cosmic infrared background fluctuations arising from clumped populations of high- $z$ infrared luminous galaxies could mimic the spectral variations from recombination line emission unless spectral resolutions $\gtrsim 5$ are available. In addition, temperature and emissivity variations of Galactic cirrus and $\mathrm{CO}$ line emission could potentially introduce excess emission in individual bandpasses that would need to be measured and removed.

The recombination line signature may be strongest in the CMB cold spots; we therefore undertake a differential analysis where we measure the strength of foreground emission in the direction of $\mathrm{CMB}$ hot spots $\left(\Delta T_{\mathrm{CMB}}>0 \mathrm{~K}\right)$ to subtract it in the direction of the $\mathrm{CMB}$ cold spots $\left(\Delta T_{\mathrm{CMB}}<0 \mathrm{~K}\right)$. Since the $\mathrm{CMB}$ temperature anisotropies are to first order, uncorrelated with the presence of foreground emission such as galaxy clusters (responsible for the Sunyaev-Zeldovich effect), cirrus emission and CIB fluctuations, the CMB hot spots should provide a robust template for eliminating all foregrounds. Although second-order effects like the late-time Sachs-Wolfe effect can result in a correlation between $\mathrm{CMB}$ cold spots and foreground overdensities, these should not produce a spectral variation across the Planck bandpasses. We describe the process of foreground cleaning in Section 2.

After cleaning the individual frequency maps of $\mathrm{CMB}$ and foregrounds, we should have noise dominated maps with the only residual emission arising from recombination line emission from $z \sim 1000$. We study the properties of the smoothed residual maps in Section 3 paying particular attention to the 143 and $353 \mathrm{GHz}$ bands where recombination line emission is expected to be the strongest. In Section 4, we assess the significance of excess seen in the residual emission and provide various scenarios to explain this excess. We summarize our conclusions in Section 5.

\section{REMOVING THE CMB AND INTERSTELLAR MEDIUM FOREGROUNDS FROM FREQUENCY MAPS}

We utilize the publicly released (PR2) Planck full-mission, full-sky, HEALPix-format, frequency maps (Górski et al. 2005) between 100 and $857 \mathrm{GHz}$. The $857 \mathrm{GHz}$ frequency map serves as a template for Galactic interstellar medium emission which is a significant contributor to the emission at high latitudes at the other frequencies (Planck Collaboration et al. 2015c). We also utilize the Commander and SMICA component-separated maps of the CMB emission (Planck Collaboration et al. 2015e).

As a first step, we mask out sources and Galactic plane emission. For this, we use as a starting point the conservative confidence mask generated for CMB component separation to mask the Galactic Plane; this is called the "UT78 mask." The mask is extended to exclude regions whose Galactic latitude is less than $20^{\circ}$. As a further extension, galaxy clusters detected through the Sunya'ev-Zeldovich from the "SZ-Union" catalog are masked out to a radius of $10^{\prime}$. Finally, for each frequency, we also mask sources from the corresponding, high-confidence, Planck catalog of compact sources (PCCS2; Planck Collaboration et al. 2015f) out to a radius of the full-width half-maximum (FWHM) at that frequency. The source mask used for the CMB map is the product of the masks for the $100-353 \mathrm{GHz}$ channels since those are the frequencies where the CMB is significant.

We convert the frequency maps at 545 and $857 \mathrm{GHz}$ to thermodynamic temperature Kelvin ${ }_{\mathrm{CMB}}$ units using the calibration factors provided in Planck Collaboration et al. (2015b); the lower frequency maps are already in these units. We then subtract the CMB map resulting from the SMICA component separation technique, from the individual frequency maps; this yields a CMB-free map at all frequencies, although the contribution from the $\mathrm{CMB}$ at 545 and $857 \mathrm{GHz}$ is negligibly small. We note that since the $\mathrm{CMB}$ is constant across all frequencies in Kelvin ${ }_{\mathrm{CMB}}$ units, no frequency dependent scaling factor is required. We then degrade the spatial resolution of the masked, CMB-free maps to 13!7 (NSIDE = 256).

We next subtract out foreground sources of emission, the dominant among which are the Galactic interstellar medium and the fluctuations due to the integrated emission from unresolved sources that contribute to the cosmic infrared background. For this purpose, we convert the CMB-free maps from the earlier step, back into $\mathrm{MJy} \mathrm{sr}^{-1}$ units, using the calibration factors provided in Planck Collaboration et al. (2015b). Since the properties of the interstellar medium vary on relatively small angular scales, we use a differential technique whereby the properties of the Galactic foregrounds at the location of the CMB hot spots within 1.5 of a CMB cold spot in the $\mathrm{CMB}$ map are used to estimate the level of foreground emission at the location of the cold spot. As a consistency check, we repeat the analysis using a 0.5 search radius as well. For each pixel corresponding to a positive CMB temperature fluctuation, we measure the ratio of intensities at that pixel, between each frequency and the $857 \mathrm{GHz}$ CMB-free map. This is effectively the ratio of Galactic foreground emission at a particular frequency to the ISM emission at $857 \mathrm{GHz}$. If the 
ratio is negative, due to noise, the corresponding pixel is masked out and neglected from the analysis. This yields ratio maps between each frequency and $857 \mathrm{GHz}$, with the ratio spatially varying across the sky due to variation in the color temperature of the foregrounds (see Appendix A). The median intensity ratios, relative to $857 \mathrm{GHz}$ in $\mathrm{MJy} \mathrm{sr}^{-1}$ units, are $0.0024,0.0078,0.033,0.14$, and 0.41 at 100, 143, 217, 353, and $545 \mathrm{GHz}$, respectively. The standard deviations of the ratios across the sky are $0.0012,0.0020,0.0067,0.020$, and 0.043 at these frequencies, respectively. The standard deviations are not the noise in the measurements but illustrate the range in colors of the ISM as illustrated in the all sky maps in Figure 4 in Appendix A. For comparison, a $17.5 \mathrm{~K}$ far-infrared color temperature blackbody with $\nu^{1.4}$ dust emissivity would show ratios of $0.0025,0.0076,0.03,0.13$, and 0.40 illustrating that the median ratios are consistent with ISM dust although with an emissivity that is somewhat lower than the canonical value of 1.6. All numbers in this paper, are derived for analysis with the SMICA CMB map subtracted, unless explicitly stated.

For each negative $\mathrm{CMB}$ temperature fluctuation i.e., a $\mathrm{CMB}$ cold spot, we then identify all pixels within 1.5 radius which correspond to a $\mathrm{CMB}$ hot spot and have a valid color ratio measured. We take the median of these pixels and use that value as the colors of the foreground emission for the $\mathrm{CMB}$ cold spot. We scale the $857 \mathrm{GHz}$ emission at that $\mathrm{CMB}$ cold spot pixel by this ratio and subtract it from the corresponding pixel at each of the frequencies. This yields a map of the sky, free of CMB fluctuations and all foregrounds that were detectable at $857 \mathrm{GHz}$ and provides the basis for further analysis. These maps have standard deviations of 0.0023 , $0.0020,0.0043,0.012$, and $0.025 \mathrm{MJy} \mathrm{sr}^{-1}$ at 100 through $545 \mathrm{GHz}$, respectively. We note that using a smaller radius of 0.5 for foreground subtraction results in noise values which are $1.06,0.98,0.92,0.9$, and 0.86 times these values. This is not surprising - at frequencies where the ISM dominates, using a small radius accounts for structure in the ISM and results in cleaner foreground subtraction while having $\sim 10 \%$ fewer pixels left for analysis. These map products are then rebinned to lower spatial resolution corresponding to pixels of 1.83 and 3 . 67 , while ignoring the pixels that are masked. It is worth noting that the footprint of the low spatial resolution pixels overlaps both CMB hot and cold spots but since the residual at the location of the hotspots at all frequencies is zero, the lowresolution maps are a measure of the residual emission at only the location of the CMB cold spots.

Upon publication, all map products as a result of this analysis will be publicly available at this website for community analysis. ${ }^{4}$

\section{SPECTRAL VARIATIONS OF THE SKY}

After subtracting out foregrounds and masking pixels which have detectable sources, the rebinned, source-masked, residual maps are free of spatial structures and should be noise dominated. Noise maps are generated by propagating the measurement uncertainties through the entire analysis procedure. For the CMB subtraction, we simply use the difference in the CMB maps generated from the Planck half-ring maps as a tracer of the uncertainty in fitting for the CMB. We note that due to the Planck scan strategy, the range of measurement noise values in the maps spans a wide range, $>40$, and depends

\footnotetext{
http://www.its.caltech.edu/ rchary/multiverse/
}

on the frequency..$^{5}$ The residual and noise maps are shown in Figure 5 in Appendix B. Dividing the residual map with a noise map yields a low-resolution signal-to-noise map. The properties of the signal-to-noise maps and residual signal are discussed in the Appendix.

Histograms of the signal to noise clearly show that although the median of the maps are zero at 217,353 , and $545 \mathrm{GHz}$, at 100 and $143 \mathrm{GHz}$, they are $\sim 2 \sigma$ offset from zero. The median intensity has a value of $1.43 \pm 0.78$ and $1.04 \pm 0.9 \mathrm{kJy} \mathrm{sr}^{-1}$ at these two frequencies, respectively, with the standard deviation measured over the unmasked sky. It can be fit with a $I_{\nu} \propto \nu^{-0.7 \pm 0.3}$ spectrum indicating that it is residual high latitude synchrotron emission which is likely uncorrelated with the diffuse ISM that was detected at $857 \mathrm{GHz}$ and which was used to subtract the foreground ISM.

Both synchrotron and free-free emission have well-defined spectral indices unlike thermal dust emission which can span a wide range of color temperatures. However, the noise in the Planck $100 \mathrm{GHz}$ maps is substantially higher than that at $143 \mathrm{GHz}$ (Table 1) which would result in a noisy residual map if we were to subtract this synchrotron emission using the $100 \mathrm{GHz}$ residual map as a template, without knowing the spatial variation in the spectral index of emission. As a result, we first identify pixels which have $\mathrm{S} / \mathrm{Ns}$ at 143 and/or $353 \mathrm{GHz}$ greater than 5 since these are the band passes where the intensity of the recombination lines from $z \sim 1000$ is thought to be the strongest. We then sub-select among these, the pixels whose residual emission at $143 \mathrm{GHz}$ is greater than $2 \sigma$ above the residual flux density at 100 and $217 \mathrm{GHz}$. That is, since we are searching for line emission in the Planck bandpasses, we require $\left(I_{143}-\sigma_{143}\right)>\left(I_{100}+\sigma_{100}\right)$ and $\left(I_{143}-\sigma_{143}\right)>\left(I_{217}+\sigma_{217}\right)$. Furthermore, due to the possibility of residual ISM contamination, we require that the ISM values lie in the lowest third of the observed range, i.e., pixels with $143 \mathrm{GHz} \mathrm{S} / \mathrm{N}>5$ fall in the range $0.8-12.5 \mathrm{MJy} \mathrm{sr}^{-1}$ in the $857 \mathrm{GHz}$ frequency map and we place an upper limit of $4 \mathrm{MJy} \mathrm{sr}^{-1}$ for the allowable range of intensity.

After eliminating edge effects, there are a total of five regions we find which meet these criteria; three are based on the analysis using the SMICA map while two are based on the analysis using the Commander CMB map (Figure 1). They are listed in Table 2. The $143 \mathrm{GHz}$ intensity in these regions is $\sim(1.3-3.2) \times 10^{-3} \mathrm{MJy} \mathrm{sr}^{-1}$ in these regions with an uncertainty of $(0.14-0.4) \times 10^{-3} \mathrm{MJy} \mathrm{sr}^{-1}$ depending on the area over which the signal is averaged (Table 2). Of these, the only region which has structure on both the $1^{\circ} .8$ and 3.6 spatial scales is the one at $(l, b)=\left(84^{\circ},-69^{\circ}\right)$ (Figure 2). The $143 \mathrm{GHz}$ residual intensity in this region is $1.3 \pm 0.14 \mathrm{kJy} \mathrm{sr}^{-1}$ at the 3.7 scale and $2.5 \pm 0.34 \mathrm{kJy} \mathrm{sr}^{-1}$ at the 1.8 scale.

Although the statistical significance of this excess is high, the noise in the adjacent bands, 100 and $217 \mathrm{GHz}$ is higher than at $143 \mathrm{GHz}$. Therefore, it is prudent to Monte-Carlo the foreground emission to assess the true statistical significance of this excess. We use a random number generator to scatter the measured residual intensities in the three bands 100, 143, and $217 \mathrm{GHz}$. The random numbers have a standard deviation which is the same as the measurement uncertainty at each frequency (Table 2). For each of the 1000 iterations, we fit the randomized 100 and $217 \mathrm{GHz}$ values with a power law to predict the $143 \mathrm{GHz}$ intensity. We compare this interpolated

\footnotetext{
5 An earlier version of this analysis neglected the spatial variation of the noise and obtained incorrect results.
} 


\section{8 deg Pixels with SNR>5 Excess at $143 \mathrm{GHz}$ and low ISM emission}

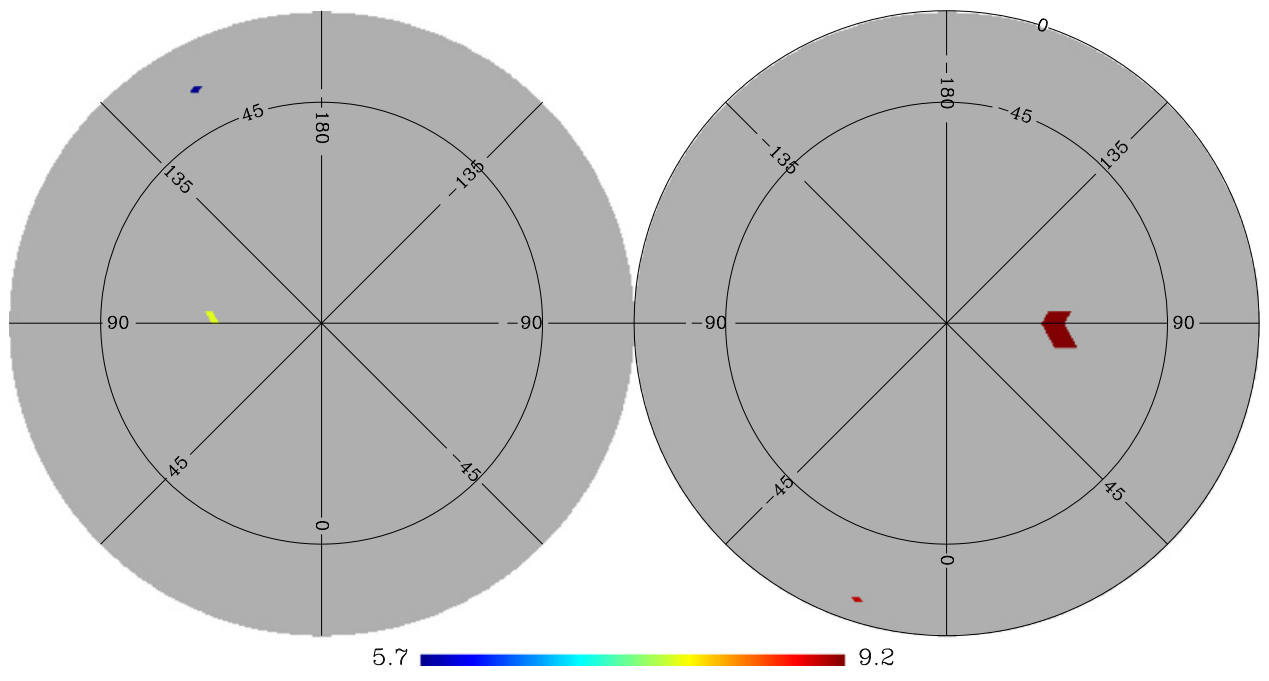

Figure 1. Galactic orthographic projections of the entire sky showing the five positions on the sky which have $\mathrm{S} / \mathrm{N}>5$ excess at $143 \mathrm{GHz}$ after foreground cleaning, and whose residual $143 \mathrm{GHz}$ intensity is more than $2 \sigma$ above the 100 and $217 \mathrm{GHz}$ intensities. All the points have $857 \mathrm{GHz}$ ISM emission less than $4 \mathrm{MJy}$ sr ${ }^{-1}$. The points are color coded by $143 \mathrm{GHz}$ signal to noise ratio. The left circle shows the northern hemisphere while the right circle shows the southern hemisphere with the Galactic equator around the circumference of the circles. The pixel scale is 1:8. Exact coordinates are given in Table 2. If this excess arises from enhanced recombination line emission at $z \sim 1000$, it would argue in favor of a collision with an alternate universe with a higher baryon to photon ratio than our own. A MonteCarlo analysis indicates that this excess is significant at the $>5 \sigma$ level $44 \%$ of the time. A simpler explanation would favor noise spikes on top of a combination of Galactic synchrotron and free-free emission.

Table 2

Multi-frequency Properties of Regions with High Reliability Excess at $143 \mathrm{GHz}$. Note that the First Two Regions are Effectively the Same ${ }^{\mathrm{a}}$

\begin{tabular}{|c|c|c|c|c|c|c|c|c|c|c|}
\hline$(l, b)$ & $\theta$ & Technique & $I_{100}$ & $\mathrm{~S} / \mathrm{N}_{143}$ & $I_{143}$ & $I_{217}$ & $\mathrm{~S} / \mathrm{N}_{353}$ & $I_{353}$ & $I_{545}$ & $I_{857}$ \\
\hline$(86.8,-69.4)$ & 1.8 & SMICA & $1.5 \pm 0.5$ & 7.3 & 2.5 & $1.3 \pm 0.7$ & -0.6 & -0.9 & $-5.9 \pm 2.9$ & 1.5 \\
\hline$(83.6,-69.4)$ & 3.7 & SMICA & $0.9 \pm 0.2$ & 9.2 & 1.3 & $0.3 \pm 0.3$ & -1.5 & -1.0 & $-2.7 \pm 1.2$ & 1.4 \\
\hline$(93.2,69.4)$ & 1.8 & SMICA & $2.2 \pm 0.5$ & 7.8 & 3.2 & $0.9 \pm 0.7$ & -0.8 & -1.5 & $-12.9 \pm 3.6$ & 1.1 \\
\hline$(151.9,31.4)$ & 1.8 & Commander & $0.8 \pm 0.3$ & 5.7 & 1.3 & $-0.1 \pm 0.5$ & -1.3 & -1.8 & $-1.3 \pm 3.1$ & 2.4 \\
\hline$(341.7,-22.0)$ & 1.8 & Commander & $1.6 \pm 0.4$ & 9.0 & 2.2 & $0.5 \pm 0.6$ & -1.4 & -1.8 & $-3.6 \pm 2.2$ & 3.2 \\
\hline
\end{tabular}

Note.

${ }^{a}$ Galactic coordinates $(l, b)$ are given in degrees, while $\theta$ is the angular resolution of the rebinned maps in degrees. Intensities are given in $\mathrm{kJy} \mathrm{sr}^{-1}$ units except for $I_{857}$ which is in $\mathrm{MJy} \mathrm{sr}^{-1}$.

intensity with the correspondingly randomized $143 \mathrm{GHz}$ intensity from the Monte-Carlo. We then find the percentage of trials where the scattered intensity is $>5 \sigma$ or $>3 \sigma$ of the interpolated $143 \mathrm{GHz}$ intensity. The percentage of such trials is listed in Table 3.

At $(l, b)=\left(84^{\circ},-69^{\circ}\right)$, the most promising region of the five selected regions where the $143 \mathrm{GHz}$ excess extends on scales of $\sim 2^{\circ}-4^{\circ}$, we find that the probability that the excess is significant at the $>3 \sigma$ level is $76 \%$ while the probability that the excess is significant at the $>5 \sigma$ level is $44 \%$. Thus, although the excess emission at $143 \mathrm{GHz}$ in those regions is $\gg 5 \sigma$ in terms of measurement uncertainty, due to contamination from synchrotron emission and the large uncertainty in the adjacent bands at 100 and $217 \mathrm{GHz}$, which makes for uncertainty in fitting the residual foreground emission, the statistical significance of the excess is substantially reduced.

Also shown in Table 3, is the signal to noise of the $143 \mathrm{GHz}$ excess $\left(\mathrm{S} / \mathrm{N}_{\text {excess,143 }}\right)$ after a power law $\left(I_{\nu} \propto \nu^{\alpha}\right)$ to the synchrotron or free-free foreground is fit to the measured residuals at 100 and $217 \mathrm{GHz}$ and interpolated to $143 \mathrm{GHz}$. This simply shows how far above the residual foregrounds the $143 \mathrm{GHz}$ excess is and does not take into account the measurement uncertainty at 100 and $217 \mathrm{GHz}$ which we have factored in the Monte-Carlo analysis. ${ }^{6}$

Finally, in order to confirm that the appearance of excess at $143 \mathrm{GHz}$ is a feature of pixels that are associated with CMB cold spots, we repeat the exercise in reverse. That is, for each pixel corresponding to a negative CMB temperature fluctuation, we measure the ratio of intensities at that pixel, between each frequency and the $857 \mathrm{GHz}$ CMB-free map. Then for each $\mathrm{CMB}$ hot spot, corresponding to an under density at the epoch of recombination, we then identify all pixels within 1.5 (or 0.5 ) radius which correspond to a $\mathrm{CMB}$ cold spot and have a valid color ratio measured. We take the median of these pixels and use that value as the colors of the foreground emission for the CMB hot spot to obtain a map which should contain all foregrounds removed at the location of the CMB hot spots. These maps are similarly rebinned to lower spatial resolution while ignoring the pixels that are masked. We find that these map products do not contain any significant evidence for excess emission at $143 \mathrm{GHz}$, indicating that under densities

\footnotetext{
6 In the Appendix, we repeated this analysis while including the Planck measurement at $30 \mathrm{GHz}$ for a stronger constraint on the foregrounds. There was little impact on the conclusion.
} 

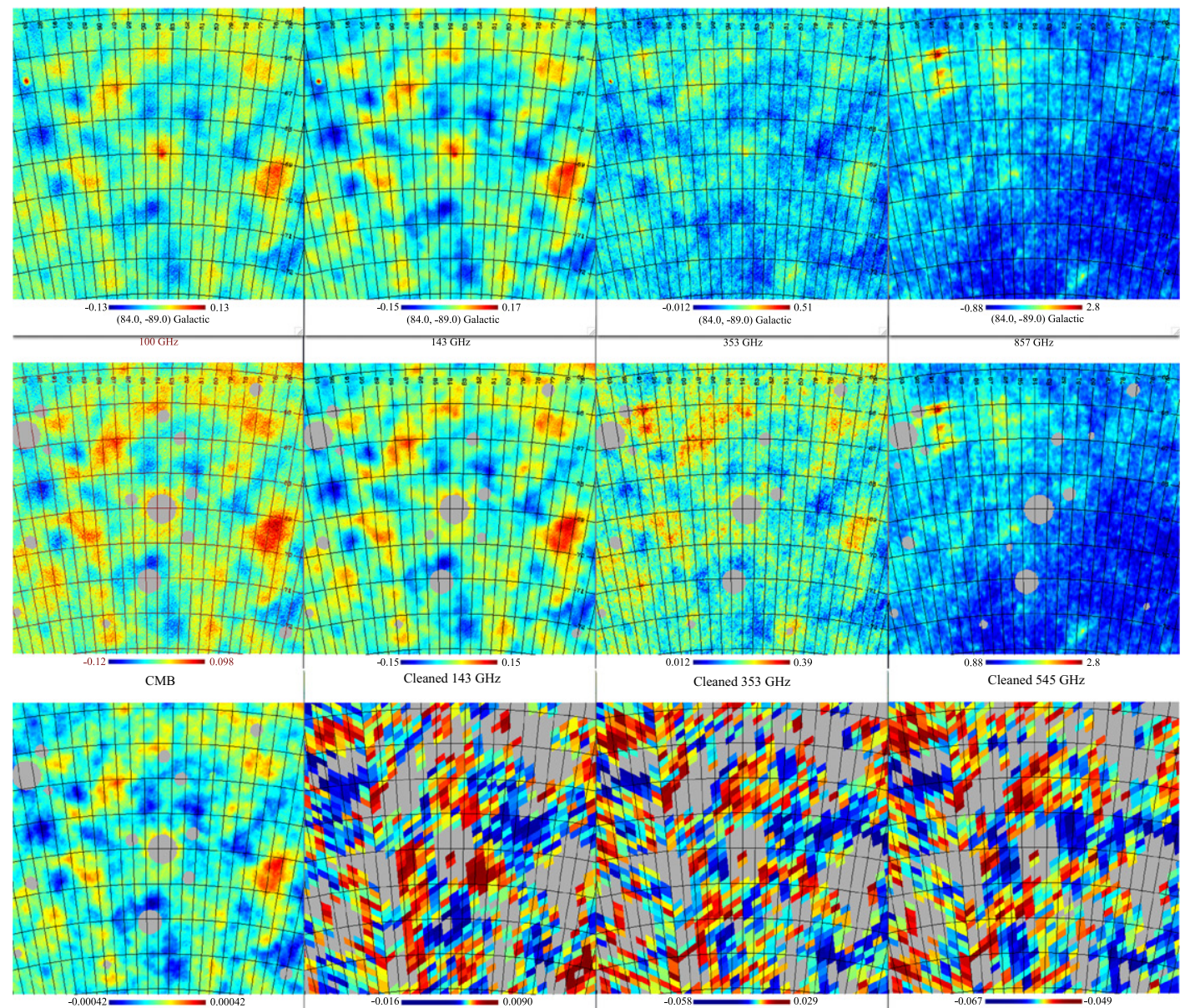

$143 \mathrm{GHz}$ Excess

$353 \mathrm{GHz}$ Excess

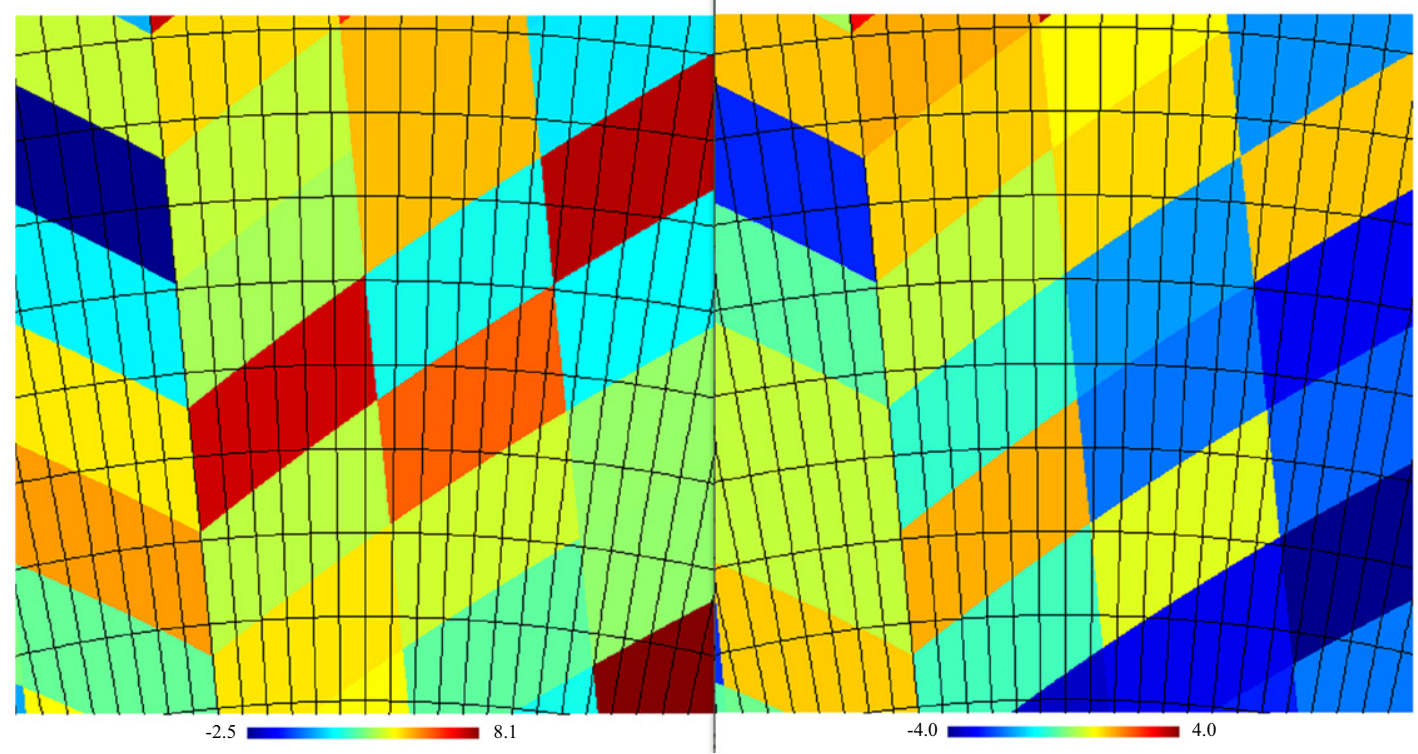

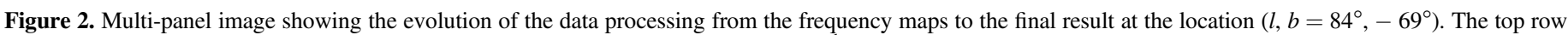

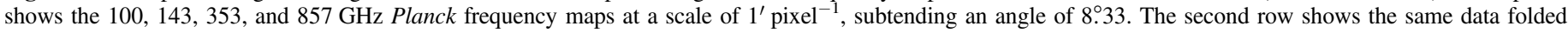

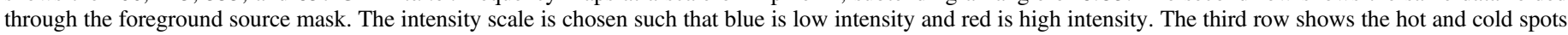

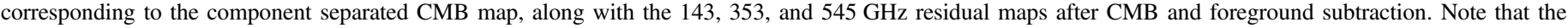

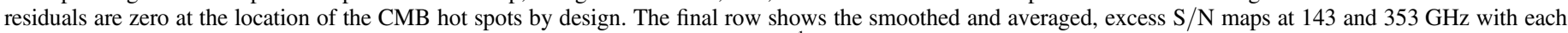

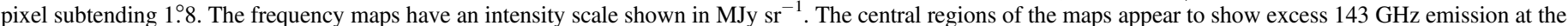
location of $\mathrm{CMB}$ cold spots that do not correspond to regions of strong ISM emission, i.e., $\ll 4 \mathrm{MJy} \mathrm{sr}^{-1}$ at $857 \mathrm{GHz}$. 
Table 3

Significance of Excess from a Monte-Carlo Analysis of Foregrounds

\begin{tabular}{lrrrc}
\hline \hline$(l, b)$ & $P_{5 \sigma}{ }^{\mathrm{a}}$ & $P_{3 \sigma}{ }^{\mathrm{b}}$ & $\alpha^{\mathrm{c}}$ & $\mathrm{S} / \mathrm{N}_{\text {excess, 143 }}{ }^{\mathrm{d}}$ \\
\hline$(83.6,-69.4)$ & 44 & 76 & -1.17 & 5.4 \\
$(86.8,-69.4)$ & 12 & 55 & -0.14 & 3.2 \\
$(93.2,69.4)$ & 27 & 70 & -1.02 & 4.3 \\
$(151.9,31.4)$ & 6 & 36 & -0.49 & 3.2 \\
$(341.7,-22.0)$ & 35 & 67 & -1.10 & 5.2 \\
\hline
\end{tabular}

Notes.

a Percent probability of the excess being significant at the $>5 \sigma$ level.

b Percent probability of the excess being significant at the $>3 \sigma$ level.

${ }^{c}$ Spectral index of the foreground emission fit to the 100 and $217 \mathrm{GHz}$ residuals.

${ }^{\mathrm{d}} \mathrm{S} / \mathrm{N}_{\text {excess, } 143}$ is the signal to noise of the residual excess at $143 \mathrm{GHz}$ after subtracting a power-law fit to the 100 and $217 \mathrm{GHz}$ residuals from Table 2 .

probed by $\mathrm{CMB}$ fluctuations do not correspond to residual excess emission. We do find evidence for significant excess at $353 \mathrm{GHz}$ but the strength of the emission correlates well with the far-infrared color temperature of the foreground emissions in the sense that regions of cooler dust with higher 545/857 intensities show stronger $353 \mathrm{GHz}$ residual emission. This implies that at $353 \mathrm{GHz}$, the contamination from cold ISM, which has substantial structure on small angular scales, is too large to make detection of epoch of recombination signals possible. At $143 \mathrm{GHz}$, however, as demonstrated above, there is significant potential to look for spectral anomalies in the direction of the CMB cold spots since the dominant foreground residual is synchrotron emission which has a well-constrained spectral shape.

\section{INTERPRETATION OF THE SPECTRAL VARIATIONS}

We have cleaned the Planck frequency maps of CMB and foreground interstellar medium emission between 100 and $545 \mathrm{GHz}$. We expected the spectral signal at the location of the CMB cold spots after smoothing to several degree angular scales to have a mean of zero and be noise dominated. Instead we have found the intensity of the residual signal at 100 and $143 \mathrm{GHz}$ in the direction of CMB cold spots, is $\sim 2 \sigma$ offset from zero over the entire sky (Figure 6 in Appendix B). Comparison with the CMB emission or strength of ISM emission shows no evidence of a correlation (Figures 7 and 8 in Appendix B). However, the spectral index of the mean residual emission between 100 and $143 \mathrm{GHz}$ can best be explained by a $I_{\nu} \propto \nu^{-0.69}$ synchrotron spectrum suggesting that it is foreground Galactic emission.

After accounting for spatial variation of the synchrotron emission using the 100 and $217 \mathrm{GHz}$ residual maps, we find that five separate regions, of which two are adjacent, show unusually strong residual emission at $143 \mathrm{GHz}$ which is in excess of emission at 100 and $217 \mathrm{GHz}$. We undertook a Monte-Carlo analysis whereby we accounted for residual foreground emission by fitting the 100,143 , and $217 \mathrm{GHz}$ residual intensity values with a single power law. The $\chi^{2}$ distribution reveals that the probability that all of this emission is due to synchrotron or free-free foregrounds is small. It is less than $0.01 \%$ for three of the regions and $0.3 \%$ for the region at $\left(151^{\circ} .9,31^{\circ} .4\right)$. However, we find a probability of $35 \%$ for the

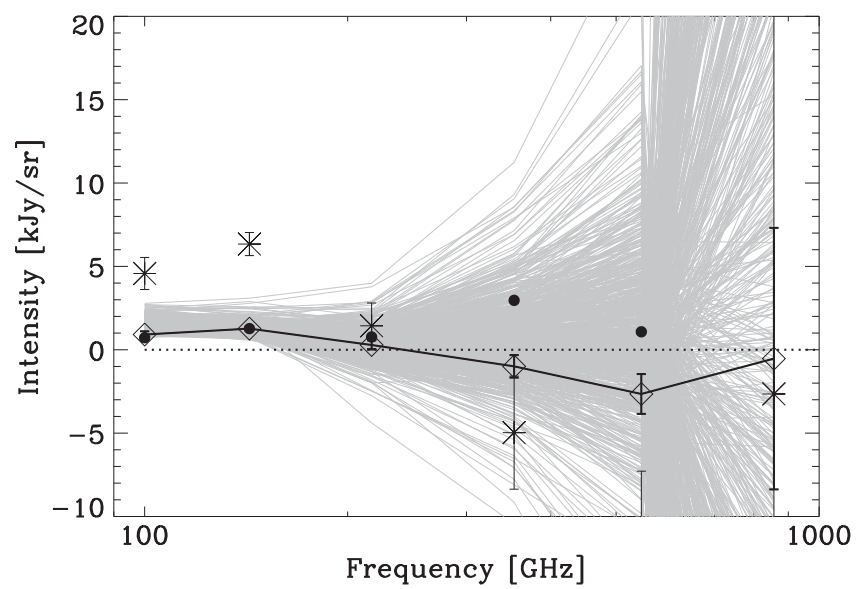

Figure 3. The thick line and diamonds indicate the spectrum of a $4^{\circ}$ spot at $\left(l, b=83^{\circ} .6,-69^{\circ} .4\right)$ where the $\mathrm{S} / \mathrm{N}$ of the residual $143 \mathrm{GHz}$ emission is greater than 5 and where the $143 \mathrm{GHz}$ emission is in excess of the residual at 100 and $217 \mathrm{GHz}$. The solid circles show a simulated spectrum of the recombination line emission using the model of Rubiño-Martín et al. (2008), Chluba et al. (2007), scaled by a factor of 4500 . The thin lines show the intensity spectrum of the cleaned sky between 100 and $545 \mathrm{GHz}$ at pixels with $143 \mathrm{GHz}$ residual $\mathrm{S} / \mathrm{N}>5$ and $857 \mathrm{GHz}$ ISM intensity less than $4 \mathrm{MJy} \mathrm{sr}^{-1}$ which would correspond to the darkest $30 \%$ Galactic ISM. The CMB and ISM emission have been removed and the resultant residual maps binned to 3.7 pixels. It is clear that the shape of the spectrum at this one location is unusual, suggestive of a contribution from strongly enhanced recombination line emission while the others can be explained by a combination of cold thermal dust emission, synchrotron, and free-free emission. The asterisks are the same residuals of the region scaled up by a factor of 5 to highlight the intensity differences between the frequencies more clearly.

region at $\left(86^{\circ} .8,-69^{\circ} .4\right)$ being fit by residual free-free emission.

Conversely, we use the Monte-Carlo to assess how large the $143 \mathrm{GHz}$ excess is relative to the emission at 100 and $217 \mathrm{GHz}$ (Table 3). We find that two of the regions are $5 \sigma$ in excess above the interpolated $100-217 \mathrm{GHz}$ spectrum $\sim 40 \%$ of the time and are $3 \sigma$ in excess almost $70 \%$ of the time. Although this is intriguing, the uncertainties in the adjacent bands at 100 and $217 \mathrm{GHz}$ are too large to provide definitive evidence for a $143 \mathrm{GHz}$ excess.

It is clear from the absence of residuals at higher frequencies that the excess is not arising due to thermal dust emission from the ISM (Figure 9 in Appendix B). This is also ruled out by the differential method undertaken to remove the foregrounds, whereby we measure the colors of the foregrounds relative to $857 \mathrm{GHz}$ at the location of the CMB hot spots to subtract the emission from the $\mathrm{CMB}$ cold spots. Calibration errors are also unlikely since the calibration accuracy in these bands is $0.1 \%$ (Planck Collaboration et al. 2015b).

A possibility based on the spectrum of residual emission is there may be a faint, Gigahertz-peaked radio source that may be unmasked or leaking flux density outside the mask. However, comparison with sources in the Planck catalog of compact sources reveals that all three radio sources in the vicinity of the region at $\left(83^{\circ} .6,-69^{\circ} .4\right)$ are synchrotron dominated sources with a spectral index $\alpha=-1.4$ where $F_{\nu} \propto \nu^{\alpha}$. The sources in the vicinity of the region at $\left(93^{\circ} .2\right.$, $69^{\circ} .4$ ) do show a peak in the spectrum at $143 \mathrm{GHz}$ with a peak flux density of $500 \mathrm{mJy}$. However, the residual emission is $3.2 \mathrm{kJy} \mathrm{sr}^{-1}$ resulting in a total flux of $3.2 \mathrm{Jy}$, much higher than the brightness of the source itself. We therefore do not think 


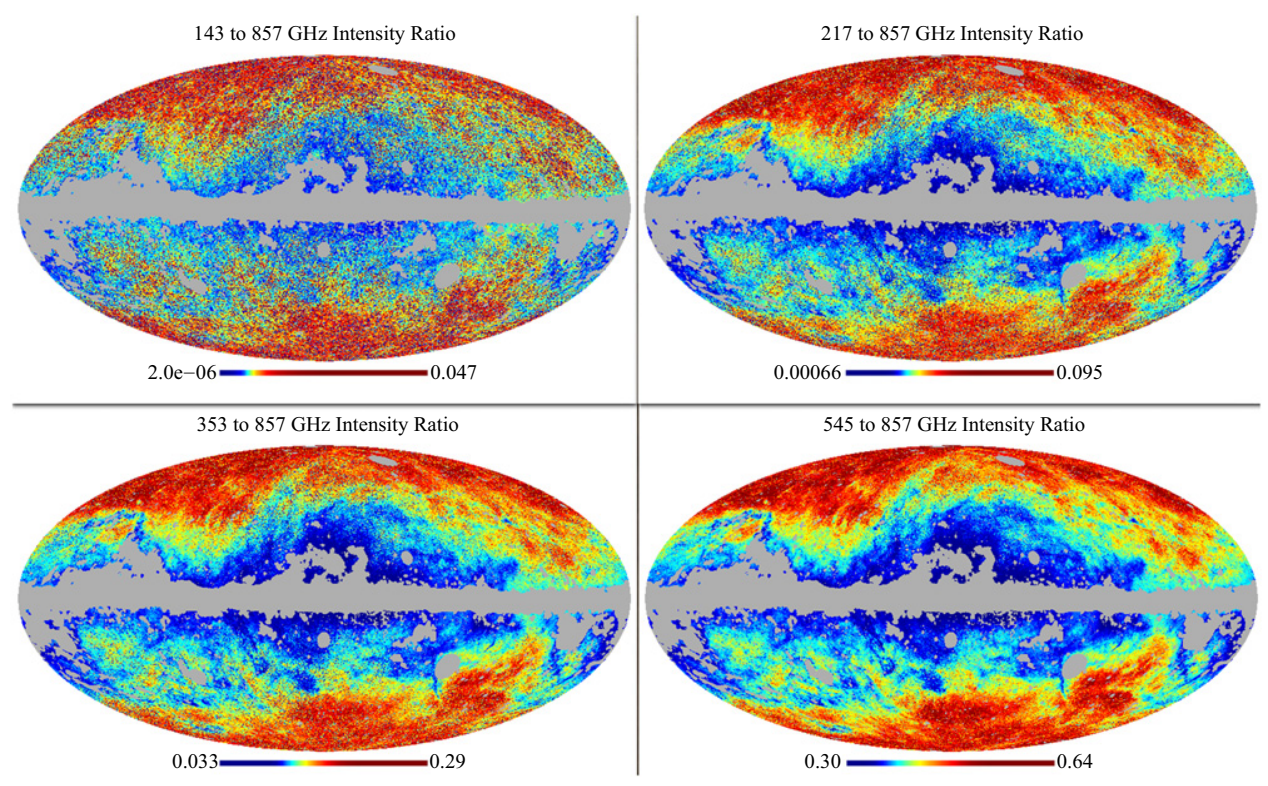

Figure 4. All-sky maps in Galactic Mollweide projection at NSIDE $=2048(1 ! 8)$ resolution showing the ratio of intensity at a particular frequency to the $857 \mathrm{GHz}$ intensity. As can be seen, higher Galactic latitudes tend to have cooler ISM emission and thereby higher ratios, than regions close to the Galactic Plane which has been masked as described in the text.

that emission leaking out from behind the source mask can account for the spectrum of this region.

Thus, if these anomalies are not extreme noise fluctuations, as analyzed above, the most likely possibilities are line emission from the ISM or cosmological/extragalactic line emission. We assess each of these in turn.

\subsection{Alternate Universes?}

We have found evidence that some of the CMB cold spots appear to have temperature decrements in the Planck $143 \mathrm{GHz}$ bandpass that are not as large as at other frequencies. These decrements, which can be detected after removing the foregrounds from the frequency maps, seem to occur primarily on angular scales of $1.8-4^{\circ}$, which is larger than the Hubble horizon at the epoch of recombination. Although we cannot conclusively rule out continuum emission of an unknown form as responsible for this excess, the absence of significantly strong excess at adjacent frequencies, and the differential method used to remove the foregrounds, suggests the contribution of line emission that is preferentially associated with the CMB cold spot.

If the lines indeed arise from the epoch of recombination, it is a factor of $\sim 4500$ enhancement of the recombination line emission compared to that expected from the models. Specifically, it would be the Paschen series hydrogen recombination lines from $z \gtrsim 1000$ that is dominating the $143 \mathrm{GHz}$ excess. Although the line in the rest-frame is emitted at $1.87 \mu \mathrm{m}$, due to the extended recombination history, in the observers frame, it spans $\sim 50 \mathrm{GHz}$ in frequency. The bandwidth of the $143 \mathrm{GHz}$ bandpass is $45.76 \mathrm{GHz}$ and therefore $2.5 \mathrm{kJy} \mathrm{sr}^{-1}$ of residual emission at $143 \mathrm{GHz}$ corresponds to a line flux of $1.1 \times 10^{-12} \mathrm{~W} \mathrm{~m}^{-2} \mathrm{sr}^{-1}$ which is about a factor of 4500 larger than expected from fiducial models for recombination line strengths from $z \sim 1000$. The clumping of baryons within the potential wells corresponding to the CMB cold spots cannot be this significant as the baryons are still thermalized, although that would be one plausible way to enhance recombination line emission. Another possibility is that the baryon density itself is enhanced by a large factor.

There is no corresponding anisotropy signal seen in the CMB temperature data. Although hints of asymmetry in the CMB power have been found with both Planck and WMAP they favor a directionality that is associated with regions of high ISM emission-regions that are excluded in our analysis (Hansen et al. 2009; Planck Collaboration et al. 2015a). This may not be surprising since the CMB intensity arises from photons while the recombination line signature arises from baryons. Since the recombination rate is proportional to the square of the baryon density for a neutral universe, but the number of Paschen- $\alpha$ photons emitted is proportional to the number of baryons, a factor of $\sim 4500$ higher baryon to photon ratio than that observed in our universe would be required to explain the signal. Since such anisotropies do not exist in our universe from what we know, a plausible explanation is that collision of our universe with an alternate universe with such a high baryon to photon ratio may be responsible for the higher recombination rate and thereby the detected signal (Aguirre \& Johnson 2011).

Collisions between bubbles with different physical properties are thought to be catastrophic events, as predicted by theory (e.g., Chang et al. 2009). They should leave an imprint on the spatial fluctuations of the CMB and typically are thought to occur prior to or during inflation. Such an event would then repeat itself across a significant fraction of sky and not be localized in space as our excess regions are. Thus, it is not clear if this interpretation has a robust theoretical framework. For the signature that we see to be due to bubble collisions but not affecting the $\mathrm{CMB}$ intensity or redshift of recombination, the collisions need to have occurred after nucleosynthesis and before matter-radiation equality when the energy density of our universe was much higher than that of the alternate universe. This could result in the injection of baryons which settled in the deeper potential wells of the density fluctuations (i.e., the cold spots) and recombined at a higher rate if these baryons are not thermalized with the CMB. Although the baryons may be 

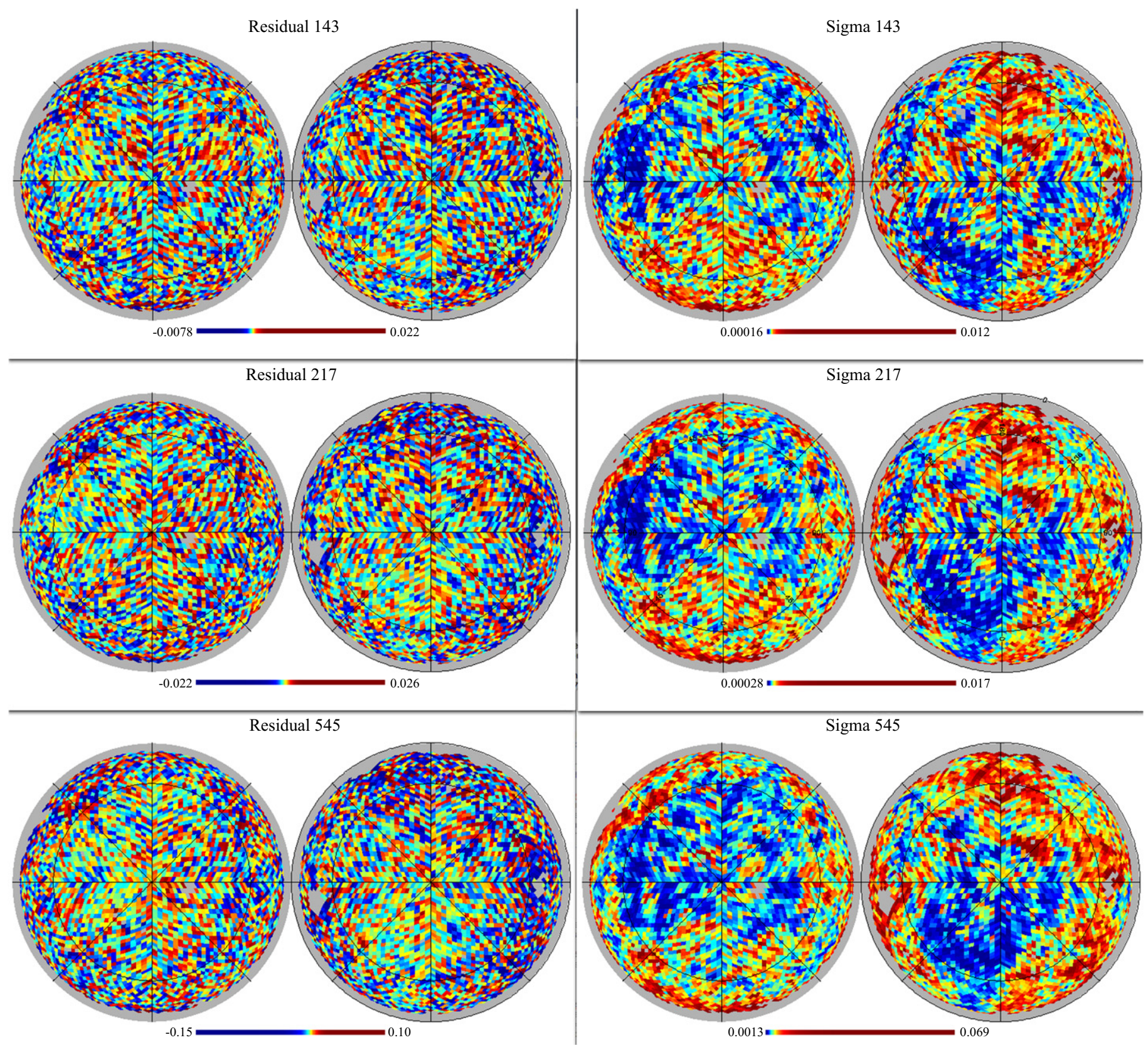

Figure 5. Left two columns shows the residual maps at 143,217 and $545 \mathrm{GHz}$ on a 1.8 pixel scale. The maps are in orthographic projection with the northern hemisphere on the left and the southern hemisphere on the right. The Galactic equator is the circumference of each circle. The Galactic center is at the bottom of the circle, the north Galactic Pole is the center of the left circle while the south Galactic Pole is the center of the right circle. The right two columns shows the noise maps at the same frequencies with the same convention. Units are $\mathrm{MJy} \mathrm{sr}^{-1}$. Due to the range of noise values resulting from the scan strategy as well as the ISM, it is incorrect to use a single noise value per frequency for the analysis. Dividing the residual map with the noise map reveals regions with highly significant excess residual emission.

recombining at a lower rate at the location of the hot spots as well, we would be insensitive to detecting this signal due to the foreground cleaning method applied. These baryons, if they are the origin of the emission, also cannot be from our universe since they would change nucleosynthesis in certain patches of our sky and affect the CMB intensity; in particular, a higher baryon-to-photon ratio would result in the production of a lot more Lithium and some ${ }^{4} \mathrm{He}$ with a decreased abundance of ${ }^{3} \mathrm{He}$ and ${ }^{2} \mathrm{H}$.

A convincing proof of the bubble collision hypothesis would lie in the measurement of an equivalent excess in the $353 \mathrm{GHz}$ bandpass where the hydrogen Balmer recombination lines may be present (Table 1 and Figure 3). The $353 \mathrm{GHz}$ band is noisier and does show $\mathrm{S} / \mathrm{N}>5$ excess at many regions with low $857 \mathrm{GHz}$ ISM emission. Furthermore, there are at least two regions where the $143 \mathrm{GHz}$ emission is stronger than the $100 \mathrm{GHz}$ emission, however we find that there is evidence for significant residual at the higher frequencies, particularly 217 and $545 \mathrm{GHz}$. In general, we find that the $353 \mathrm{GHz}$ bandpass is more affected by cold residual ISM emission which is prevalent in our Galaxy and not perfectly traced by the $857 \mathrm{GHz}$ emission, as seen by the range of residual intensities shown in 


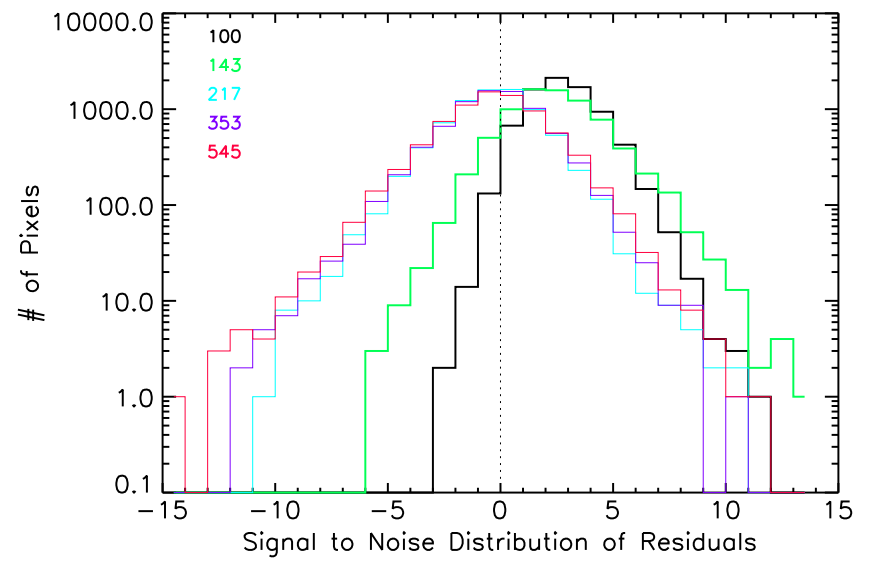

Figure 6. Histograms of the residual emission in the cleaned 1.8 resolution maps at the location of CMB cold spots at each of the Planck frequencies. Only the 100 and $143 \mathrm{GHz}$ bands show evidence for excess emission, with the median excess consistent with Galactic synchrotron radiation with a $I_{\nu} \propto \nu^{-0.69}$ spectrum.

Figure 3. Thus, we are unable to confirm the presence of excess in that band arising from the Balmer lines although we note that the predicted excess at $353 \mathrm{GHz}$ from a rescaled model, is only $\sim 2 \sigma$ offset from the measured limit.

Chluba \& Sunyaev (2009) have also demonstrated that injection of energy through dark matter annihilation in the prerecombination universe may enhance the spectral distortions arising from recombination line emission. These distortions can however be broad, spanning several tens of $\mathrm{GHz}$, and depend on the redshift at which the energy is injected and the magnitude of the injected energy. They should as a result straddle multiple Planck bandpasses which is unlike the excess that we are seeing. Without knowing the shape of the spectral distortion across the $143 \mathrm{GHz}$ bandpass, it is not possible to distinguish between these scenarios and pure recombination line emission. However, future work will attempt to combine the low-frequency Planck channels and WMAP data to assess the contribution of the weaker hydrogen Brackett and Pfundseries recombination lines to the measured intensity although a first estimate suggests the low-frequency data are too noisy to provide useful constraints.

\subsection{Foreground Interstellar Medium?}

An alternate, less exotic possibility is that the excess emission we are seeing is due to anomalous microwave emission or line contamination from the ISM. In particular, there appears to be a region of significant ISM several degrees away from the excess (Figure 2). Despite the extensive masking done to the foreground sources, diffuse line emission in the outskirts could contribute to the excess emission seen at $143 \mathrm{GHz}$. However, the $143 \mathrm{GHz}$ does not straddle energetically significant CO lines and it is unclear if some unknown sub millimeter lines associated with the star-forming regions may be contributing to the excess. Examples of such lines $\left(\mathrm{CS} 3 \rightarrow 2, \mathrm{CH}_{3} \mathrm{OCH}_{3}\right.$ ) have indeed been observed previously in star-forming regions (Öberg et al. 2010) and since they arise in the lukewarm envelope surrounding star-forming regions, they could be more extended that the thermal dust emission which has been masked. Since the thermal dust emission has an intensity of $<4 \mathrm{MJy} \mathrm{sr}^{-1}$ at this location, the ISM line emission must be over represented with respect to the thermal dust emission which is surprising since these lines are weak.

Continuum contamination from the ISM is also a possibility. The far-infrared color temperature of the emission must be as cold as $\sim 3 \mathrm{~K}$ to fit the $143 \mathrm{GHz}$ excess (Figure 9 in Appendix B). This would be the coldest dust yet detected and it would presumably be in thermal equilibrium with the CMB which is a challenge in the radiation field of our Galaxy. Such cold continuum emission, if it arises from either residual CMB or cold ISM, would violate the limits we have at $217 \mathrm{GHz}$, be uncorrelated with the warm ISM and be clumped on small scales preferentially in the direction of CMB cold spots which seems like a contrived explanation.

Finally, an analysis of star-forming regions has shown that anomalous microwave emission, which arguably arises from spinning dust grains, does not extend to frequencies as high as $143 \mathrm{GHz}$ (Planck Collaboration et al. 2014a).

Although we cannot definitively disprove the ISM contamination hypothesis, the combination of low ISM foregrounds in our regions, combined with a lack of excess at other frequencies (e.g., 100, 217, and $353 \mathrm{GHz}$ ) where the ISM is known to display strong CO lines, argues against this possibility.

\section{3. [C II] Emission from Galaxies?}

A third possibility for the $143 \mathrm{GHz}$ excess is line emission from clumped extragalactic sources. The strongest line arising in galaxies that could fall in this regime is the $\left[\mathrm{C}_{\mathrm{II}}\right]$ line at $158 \mu \mathrm{m}$, redshifted from $10<z<15$. Although the strength of [C II] in reionization epoch Lyman-break galaxies is unclear, one can make optimistic assumptions about its strength and provide an order of magnitude estimate to its contribution. The ultraviolet measured star formation rate density at $z \sim 10$ is generally thought to be around $10^{-2.5} M_{\odot} \mathrm{yr}^{-1} \mathrm{Mpc}^{-3}$ (Oesch et al. 2015). Assuming that this measurement accurately reflects the bolometric luminosity density from galaxies at these redshifts, and adopting a $[\mathrm{C} \mathrm{II}] / L_{\mathrm{Bol}}$ ratio of $\sim 10^{-2}$ which is at the high end of what has been measured for low-metallicity dwarf galaxies in the local universe (Cormier et al. 2015), we find that the total contribution from $\left[\mathrm{C}_{\mathrm{II}}\right]$ line emission in the $143 \mathrm{GHz}$ band is at most $1.7 \times 10^{-14} \mathrm{~W} \mathrm{~m}^{-2} \mathrm{sr}^{-1}$. This is almost two orders of magnitude lower than the excess we measure. Thus, it is extremely unlikely that star-forming galaxies can account for the $143 \mathrm{GHz}$ residual excess.

The broad bandpass of the Planck HFI instrument does not allow us to use the spectral profile of the lines to definitively distinguish between these scenarios and unusual claims like evidence for alternate universes require a very high burden of proof. We outline pathways for the future in the next section which could help confirm the alternate universe hypothesis.

\subsection{Future Directions}

The detection of spectral anomalies on the sky at CMB cold spots is a highly surprising result and requires further data for confirmation. It would be prudent to generate multiple realizations of the Planck frequency maps with artificial spectral distortion signals injected in the sky models. Analyzing those simulated maps using the methodology adopted here will be illustrative but is beyond the supercomputing resources that currently are available within the collaboration. The only simulations that exist are multiple realizations of the CMB sky 

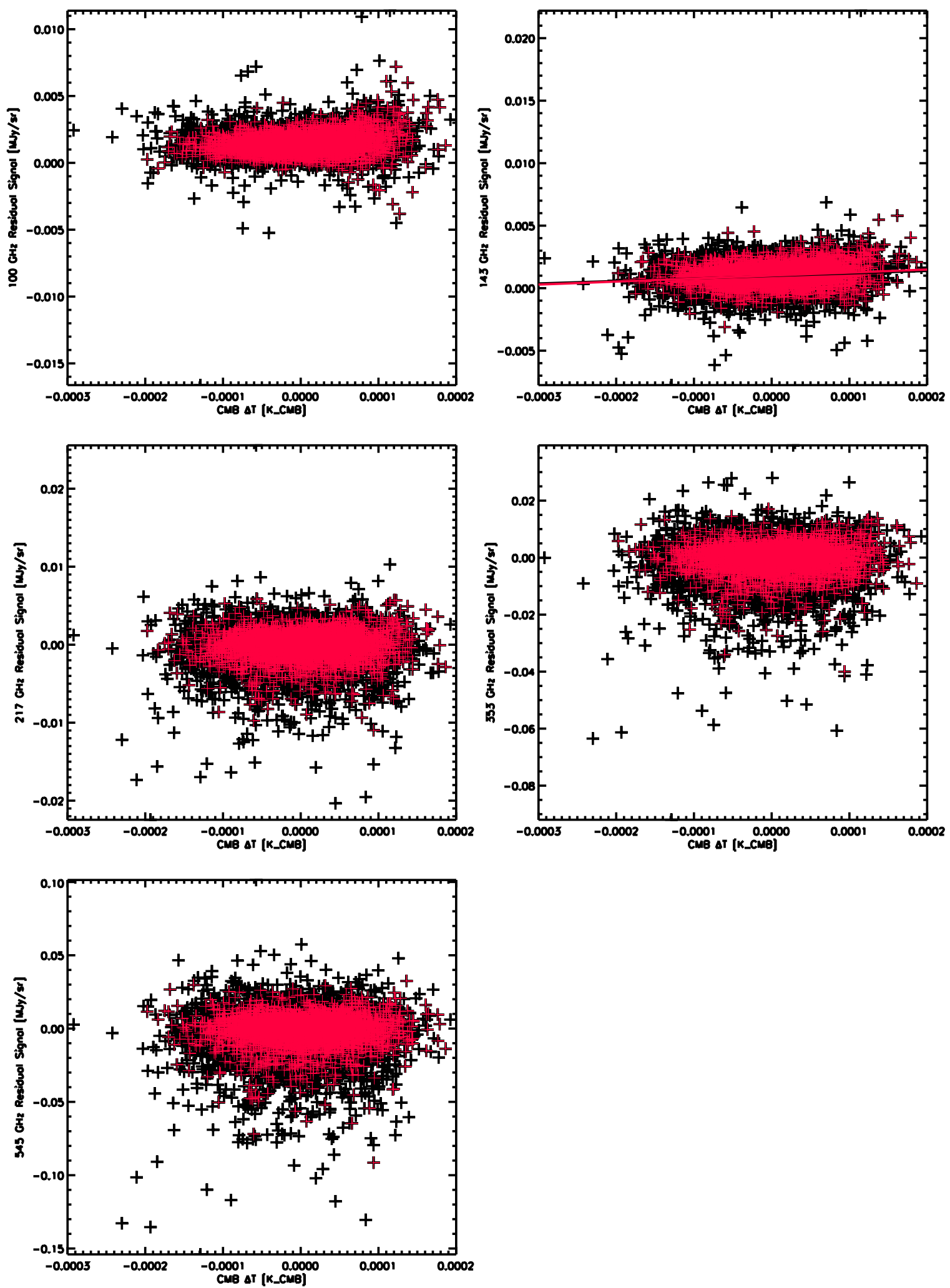

Figure 7. Residual emission at each of the frequencies shown against the CMB temperature decrement. The black points are each pixel in the 1.8 resolution maps with ISM $<8 \mathrm{MJy} \mathrm{sr}^{-1}$. The red points are only the ones with ISM $<4 \mathrm{MJy} \mathrm{sr}^{-1}$ and at Galactic latitudes greater than $45^{\circ}$. Except for 100 and $143 \mathrm{GHz}$, the median of the residuals at each frequency is consistent with zero.

but these are less useful since we subtract the same CMB map from each of the frequencies in our analysis. Such a simulation will help define the reliability with which Planck can detect such spectral anomalies.
LMT, GBT, and ALMA spectral observations in the regions with anomalous excess can help place constraints on the contribution of weak ISM lines to the $143 \mathrm{GHz}$ band intensity. Deep continuum observations at 100 and $220 \mathrm{GHz}$ of these 

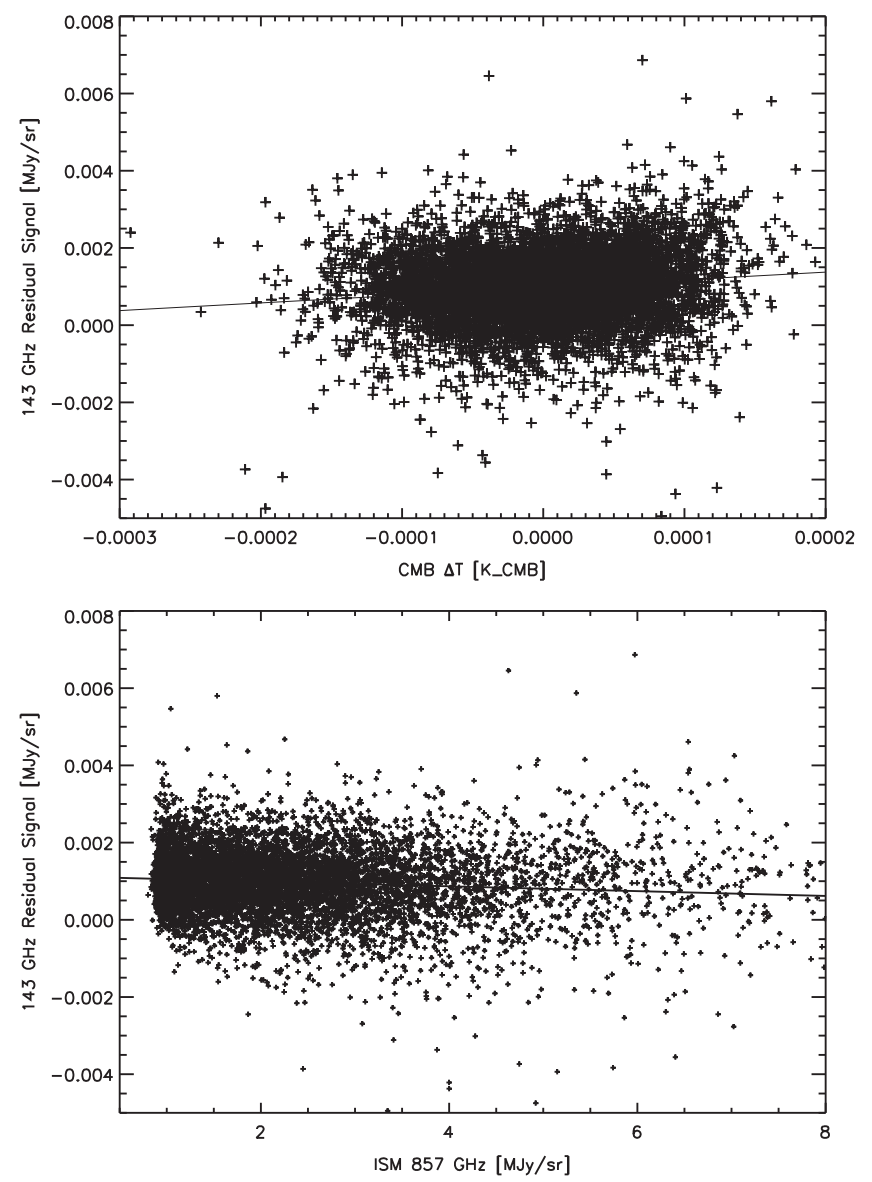

Figure 8. (Top) The strength of the $143 \mathrm{GHz}$ residual signal in the 1.8 resolution maps plotted against the temperature in the correspondingly degraded CMB map with the solid line showing the best fit. (Bottom) The strength of the $143 \mathrm{GHz}$ residual signal from CMB cold spots in the 1.8 resolution maps correlated against the $857 \mathrm{GHz}$ intensity for all pixels with ISM $<8 \mathrm{MJy} \mathrm{sr}^{-1}$. There is no evidence for a correlation here, indicating that the ISM has been well removed. However, as described in the text, the residual emission is dominated by synchrotron emission.

regions with ground-based CMB experiments such as SPT and BICEP/Keck can place tighter constraints on the synchrotron and free-free foregrounds. However, since the redshifted hydrogen Paschen- $\alpha$ recombination line falls in the middle of the atmospheric oxygen absorption line at $\sim 116-120 \mathrm{GHz}$, spectral confirmation of this signal from the ground is likely to remain challenging. Space-based CMB spectral distortion observations, such as with the Primordial Inflation Explorer (PIXIE), may provide the only way to conclusively test this hypothesis with special care given to sensitivity requirements such that key foregrounds are detected in a majority of the narrow bands (Kogut et al. 2014). Specifically, we suggest that future $\mathrm{CMB}$ missions should focus on moderate spectral resolution $(R \sim 10)$, multi-band spectroscopy achieving $<0.1 \mathrm{kJy} \mathrm{sr}^{-1}$ sensitivity, over degree scales of sky to probe for differences in fine structure constants and recombination line strengths in the early universe.

\section{CONCLUSIONS}

The CMB power spectrum extracted from the Planck frequency maps has been shown to be consistent with a $\Lambda \mathrm{CDM}$ cosmology with a specific set of cosmological parameters measured with unprecedented sensitivity (Planck
Collaboration et al. 2015d). Much of this consistency arises from precise measurements of the power spectrum on angular scales smaller than $1^{\circ}$. Why these parameters are the values they are is a question that does not have a clear answer. One possibility is there are an infinite set of universes with different parameters and our universe just happens to have the values that we measure. Searching for these alternate universes is a challenge. One hypothesis suggests that as each universe evolves independently, it may collide with our observable universe, leaving a signature on the signal we see. Since the CMB intensity has been shown to be isotropic, it is clear that such a collision is not seen in the intensity of the photons. Probing the density of baryons during the epoch of recombination however provides an alternate approach. If the baryon density is higher in these alternate universes, recombination between baryons at redshift of $>1000$ can leave signatures of hydrogen recombination line emission which are redshifted into the Planck bandpasses at 143 and $353 \mathrm{GHz}$. We have cleaned the Planck maps at all frequencies between 100 and $545 \mathrm{GHz}$ of $\mathrm{CMB}$ and foreground emission to search for this recombination line signature. We find evidence for a $2 \sigma$ offset from zero in the mean residual intensity at high latitudes at 100 and $143 \mathrm{GHz}$ which is due to residual synchrotron emission in the direction of the CMB cold spots that is uncorrelated with the $857 \mathrm{GHz}$ ISM template used to clean foregrounds. We also find a $3.2-5.4 \sigma$ excess at $143 \mathrm{GHz}$ on $1.8-3^{\circ} .7$ angular scales, after cleaning, which arises at the location of four CMB cold spots. Since the CMB cold spots are regions of over densities in the primordial density field, it appears that enhanced recombination line emission could arise from these over densities. However, the magnitude of enhancement is 4500 times higher than what is expected for the baryon density in our universe. A possible implication is that collision of our universe with an alternate universe that has a higher baryon density is responsible for the enhanced recombination line signature with a line flux of $1.1 \times 10^{-12} \mathrm{~W} \mathrm{~m}^{-2} \mathrm{sr}^{-1}$. A more prosaic explanation using a Monte-Carlo analysis suggests that Galactic synchrotron and free-free emission scattered by measurement noise could be within $3 \sigma$ of such significant excess $30 \%$ of the time; yet we find that it cannot explain all the excess. We assess possible alternate reasons for this highly significant enhancement, including the contribution of $\mathrm{CO}$ and other lines in the bandpass, and find that they are somewhat contrived but which can only be assessed by taking spectral data. Furthermore, if they are indeed ISM foregrounds, it does not explain why the enhanced residual emission would preferentially occur in the direction of the CMB cold spots and not in the direction of the CMB hot spots or hide itself at frequencies where the ISM is known to have stronger lines.

Planck (http://www.esa.int/Planck) is a project of the European Space Agency (ESA) with instruments provided by two scientific consortia funded by ESA member states and led by Principal Investigators from France and Italy, telescope reflectors provided through a collaboration between ESA and a scientific consortium led and funded by Denmark, and additional contributions from NASA (USA). High quality data products have been generated through extensive work by the HFI Core Team lead by J.-L. Puget and the Planck Collaboration as a whole. The author wishes to thanks A. Banday, G. Helou, M. Kleban, E. Wright, J. Chluba and J. Carpenter for feedback and discussions and G. Fazio and 

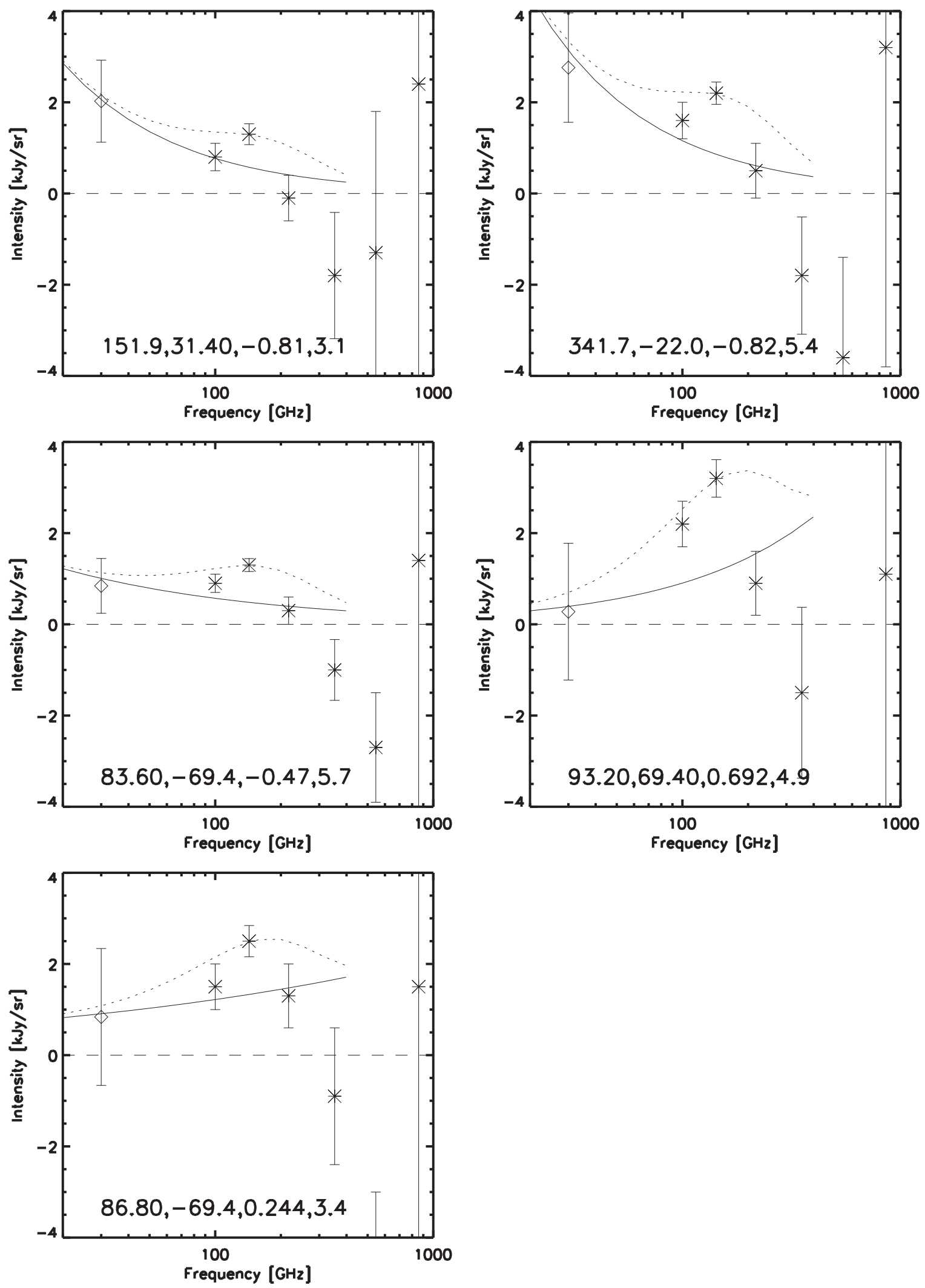

Figure 9. Five panels show as asterisks the spectrum of emission in the five regions in Table 2. Also shown (diamond) is the residual $30 \mathrm{GHz}$ intensity from the Planck LFI maps of the same resolution. The solid line is the best fit to the 30,100 , and $217 \mathrm{GHz}$ points with the four numbers at the bottom left being $\left(l, b, \alpha, \mathrm{S} / \mathrm{N}_{\text {excess, 143 }}\right)$ where $\alpha$ is the spectral index of the fit and $\mathrm{S} / \mathrm{N}_{\text {excess, } 143}$ is as defined in Table 3 . While the $3 \sigma$ points have a random probability of occurring a handful of times in a map with 12,000 pixels, the $\gtrsim 5 \sigma$ points should occur only once in 70 maps and the $5.7 \sigma$ point once in 70,000 maps. The dotted line shows the result of adding a continuum blackbody component of $2.7 \mathrm{~K}$, which may be due to residual CMB or ISM; the measurements at 100 and $217 \mathrm{GHz}$ are clearly violated, arguing that the excess is more likely due to line emission. 
J. Robie for their hospitality. This work has made use of NASA's Infrared Science Archive.

\section{APPENDIX A FOREGROUND RATIO MAPS}

In this appendix, we show the intensity ratio maps of foreground emission that are generated from the CMB-free allsky maps (Figure 4). We note that the ratios are measured at the location of the $\mathrm{CMB}$ hot spots identified in the componentseparated CMB map and then applied to the CMB cold spots as described in the text.

\section{APPENDIX B \\ PROPERTIES OF RESIDUALS AT EACH FREQUENCY}

After cleaning the individual frequency maps of CMB and foreground emission, the maps have a residual intensity with some scatter at the location of the CMB cold spots. At the location of the hot spots, the residual intensity is zero by design. These high-resolution maps are then re-binned to spatial resolutions of 1.8 and 3.7 which averages over the residual emission in a large number of $\mathrm{CMB}$ cold spots and reduces the noise in the measurement (Figure 5). Of all the frequencies, only 100 and $143 \mathrm{GHz}$ show a clear positive bias in the residual signal with the median of the distribution located at $2 \sigma$. The other frequencies have a median of zero (Figure 6). The residual intensities are uncorrelated with either the ISM emission or the CMB (Figures 7 and 8). However, the spectrum of the residual is consistent with that of Galactic synchrotron emission.

After fitting for Galactic synchrotron emission, including $30 \mathrm{GHz}$ Planck data which has been similarly cleaned of CMB fluctuations and sources, we find the residuals at $143 \mathrm{GHz}$ continue to exceed the foregrounds by $3.1-5.7 \sigma$ (Figure 9). Although the $\sim 3 \sigma$ fluctuations could occur by chance a handful of times in a map with $\sim 12,000$ pixels, the $\gtrsim 5 \sigma$ excess points in 3 of the regions have a negligible chance of occurring by random. It must therefore be due to an additional component of emission as discussed in the text.

\section{REFERENCES}

Aguirre, A., \& Johnson, M. C. 2011, RPPh, 74, 074901

Aguirre, A., \& Tegmark, M. 2005, JCAP, 1, 003

Ali-Haïmoud, Y. 2013, PhRvD, 87, 023526

Ali-Haïmoud, Y., \& Hirata, C. M. 2011, PhRvD, 83, 043513

Chang, S., Kleban, M., \& Levi, T. S. 2009, JCAP, 4, 025

Chluba, J., Rubiño-Martín, J. A., \& Sunyaev, R. A. 2007, MNRAS, 374, 1310

Chluba, J., \& Sunyaev, R. A. 2006, A\&A, 458, L29

Chluba, J., \& Sunyaev, R. A. 2009, A\&A, 501, 29

Cormier, D., Madden, S. C., Lebouteiller, V., et al. 2015, A\&A, 578, A53

Dai, L., Jeong, D., Kamionkowski, M., \& Chluba, J. 2013, PhRvD, 87, 123005

Desjacques, V., Chluba, J., Silk, J., de Bernardis, F., \& Doré, O. 2015, arXiv:1503.05589

Feeney, S. M., Johnson, M. C., Mortlock, D. J., \& Peiris, H. V. 2011, PhRvD, 84,043507

Górski, K. M., Hivon, E., Banday, A. J., et al. 2005, ApJ, 622, 759

Hansen, F. K., Banday, A. J., Górski, K. M., Eriksen, H. K., \& Lilje, P. B. 2009, ApJ, 704, 1448

Kogut, A., Chuss, D. T., Dotson, J., et al. 2014, Proc. SPIE, 9143, 91431E

Lamarre, J.-M., Puget, J.-L., Ade, P. A. R., et al. 2010, A\&A, 520, A9

Mather, J. C., Cheng, E. S., Cottingham, D. A., et al. 1994, ApJ, 420, 439

Öberg, K. I., Bottinelli, S., Jørgensen, J. K., \& van Dishoeck, E. F. 2010, ApJ, 716,825

Oesch, P. A., Bouwens, R. J., Illingworth, G. D., et al. 2015, ApJ, 808, 104

Planck Collaboration, Ade, P. A. R., Aghanim, N., et al. 2014a, A\&A, 565, A103

Planck Collaboration et al. 2014b, A\&A, 571, A1

Planck Collaboration et al. 2014c, A\&A, 571, A9

Planck Collaboration et al. 2014d, A\&A, 571, A13

Planck Collaboration et al. 2015a, A\&A, submitted (arXiv:1506.07135)

Planck Collaboration et al. 2015b, A\&A, submitted (arXiv:1502.01587)

Planck Collaboration et al. 2015c, A\&A, submitted (arXiv:1502.01588)

Planck Collaboration et al. 2015d, A\&A, submitted (arXiv:1502.01589)

Planck Collaboration et al. 2015e, A\&A, submitted (arXiv:1502.05956)

Planck Collaboration et al. 2015f, A\&A, submitted (arXiv:1507.02058)

Rubiño-Martín, J. A., Chluba, J., \& Sunyaev, R. A. 2008, A\&A, 485, 377

Sachs, R. K., \& Wolfe, A. M. 1967, ApJ, 147, 73 